Finally, we indicate a particularly interesting feature of the TST activation energy described above, that it represents an upper bound to the exact value. Note first that the exact rate constant is given by the equation

$$
k=\kappa k_{\mathrm{TST}},
$$

where $k$ is the adatom sticking probability. Substitution of $k$ into Eq. (2) therefore yields an expression for the exact activation energy

$$
\begin{aligned}
E_{a} & =\frac{k_{B} T^{2}}{\kappa k_{\mathrm{TST}}} \frac{\partial}{\partial T}\left(\kappa k_{\mathrm{TST}}\right) \\
& =\frac{k_{B} T^{2}}{k_{\mathrm{TST}}} \frac{\partial k_{\mathrm{TST}}}{\partial T}+\frac{k_{B} T^{2}}{\kappa} \frac{\partial \kappa}{\partial T} \\
& =E_{a}^{\mathrm{TST}}+\frac{k_{B} T^{2}}{\kappa} \frac{\partial \kappa}{\partial T} .
\end{aligned}
$$

However, since for a simple desorptive event the sticking probability is likely to be a nonincreasing function of the temperature, one may conclude that for such simple processes

$$
E_{a} \leq E_{a}^{\mathrm{TST}} \text {. }
$$

(Since $k \leq k_{\text {TST }}$, the above condition means that the desorption frequency factor calculated within the TST formalism must also provide an upper bound.) of course, such a bounding argument would be of little value if it were not for the fact that the activation energy can be computed to arbitrarily good accuracy via the Monte Carlo method.

Although in our earlier paper ${ }^{1}$ we suggested that the synthesis of transition state theory and pseudodynamical Monte Carlo techniques would prove to be a singularly useful means of obtaining desorption rate data, it now appears clear that the inclusion of importance sampling makes this approach even more powerful. The method does, for example, overcome the usual problems associated with the simulation of intrinsically rare events, ${ }^{6}$ while at the same time permitting the study of the adatom concentration effects which are practically inaccessible by other methods. It is, in fact, this rather unique versatility of the Monte Carlo-TST formalism which most strongly commends itself to those interested in investigating adsorption/desorption phenomena.

a) Work supported by the U. S. Department of Energy.

1J. E. Adams and J. D. Doll, J. Chem. Phys. 74, 1467 (1981).

${ }^{2}$ J. E. Adams and J. D. Doll, Surf. Sci. (in press).

${ }^{3}$ D. Chandler, J. Chem. Phys. 68, 2959 (1978).

${ }^{4}$ See, for example, J. P. Valleau and S. G. Whittington, in Statistical Mechanics; Part A, edited by B. J. Berne (Plenum, New York, 1977).

${ }^{5}$ R. C. Tolman, J. Am. Chem. Soc, 42, $2506(1920) ; 47,2652$ (1925).

${ }^{6}$ E. K. Grimmelmann, J. C. Tully, and E. Helfand, J. Chem. Phys. 74, 5300 (1981).

\title{
Erratum: Bispectral analysis as a probe of quasielastic light scattering intensity fluctuations [J. Chem. Phys. 72, 6123 (1980)]
}

\author{
George D. J. Phillies \\ Department of Chemistry, The University of Michigan, Ann Arbor, Michigan 48109
}

In a recent paper, this author suggested that the measurement of the homodyne bispectrum $\langle I(0) I(t) I(\tau)\rangle$ might be of use in interpreting light scattering spectra of solutions containing several noninteracting diffusing species, in that the second time cumulants of the bispectrum were

$$
\begin{aligned}
K_{02}= & K_{20}=\sum_{i, j} \bar{a}_{i}^{2} \bar{a}_{j}^{2}\left(\Gamma_{l}-\Gamma_{j}\right)^{2} /\left(\sum_{m} \bar{a}_{m}^{2}\right)^{2}, \\
K_{11}= & {\left[-\sum_{i, j} \bar{a}_{i}^{4} \bar{a}_{j}^{2}\left(\Gamma_{i}-\Gamma_{j}\right)^{2}\right.} \\
& \left.\times \sum_{i \neq j \neq k \neq i} \bar{a}_{i}^{2} \bar{a}_{j}^{2} \bar{a}_{k}^{2}\left(\Gamma_{j}-\Gamma_{i}\right)\left(\Gamma_{j}+\Gamma_{k}\right)\right] /\left(\sum_{m} \bar{a}_{m}^{2}\right)^{3},
\end{aligned}
$$

where $\bar{a}_{i}$ and $\Gamma_{i}$ are the root-mean-square scattering amplitudes and the decay rate, respectively, for species $i$. For a system containing two diffusing species, $K_{11}$ $=\frac{1}{2} K_{02}$; for a system containing more than two species, $K_{11}$ and $K_{02}$ do not appear to be related by a simple constant.

Contrary to the appearance remarked upon in Ref. 1, it is possible to manipulate Eq. (1b) into a form which is simply related to Eq. (1a), namely,

$$
\begin{aligned}
K_{11}= & {\left[-\frac{1}{2} \sum_{i, j} \bar{a}_{i}^{2} \bar{a}_{j}^{2}\left(\Gamma_{i}-\Gamma_{j}\right)^{2}\left(\bar{a}_{i}^{2}+\bar{a}_{j}^{2}\right)\right.} \\
& \left.-\frac{1}{2} \sum_{i \neq j \neq k \neq i} \bar{a}_{j}^{2} \bar{a}_{i}^{2}\left(2 \Gamma_{j}^{2}-2 \Gamma_{j} \Gamma_{i}\right) \bar{a}_{k}^{2}\right] /\left(\sum_{l} \bar{a}_{i}^{2}\right)^{3} ;
\end{aligned}
$$


the symmetry of the final term under $i \rightarrow j$ lets one write

$$
K_{11}=-\frac{1}{2} \sum_{i, j}\left[\bar{a}_{i}^{2} \bar{a}_{j}^{2}\left(\Gamma_{i}-\Gamma_{j}\right)^{2}\right]\left(\sum_{k} \bar{a}_{k}^{2}\right) /\left(\sum_{i} \bar{a}_{l}^{2}\right)^{3}
$$

so that for an arbitrary number of solute species $K_{1}$ : $=-\frac{1}{2} K_{02}=-\frac{1}{2} K_{20}$. While the results of Ref. 1 are mathematically correct, the conclusion of Ref. 1 , that bispectral analysis permits one to distinguish between systems characterized by two or by more than two decay times, is not supported by the mathematical results in that reference.

\section{Erratum: On the quantitation of light emission from cytochrome $c$ in the low quantum yield limit [J. Chem. Phys. 73, 5947 (1980)]}

P. M. Champion a) and R. Lange

Institut de Biologie Physico-Chimique, Fondation Edmond de Rothschild, Service de Biospectroscopie, 75005, Paris, France

and Institut National de la Sante et de la Researche Medicale, U 128 B.P. 5051, 34033 Montpellier, France

On p. 5956, right column, line 25 , change 50 psec to $50 \mathrm{fsec}$.

3) Present address: Laboratory of Atomic and Solid State Physics, Cornell University, Ithaca, N. Y. 14853. 